\title{
Predictive value of incidental right ventricular abnormalities identified on SPECT for mortality and pulmonary hypertension
}

\author{
Arun Jose, MD, MS, ${ }^{\mathrm{a}}$ Christine Zhou, DO, ${ }^{\mathrm{b}}$ Rachel Baker, ${ }^{\mathrm{c}}$ Jackson Walker, MD, ${ }^{\mathrm{b}}$ \\ Nicholas Kurek, MD, ${ }^{\mathrm{b}}$ Robert E. O'Donnell, MD, ${ }^{\mathrm{d}}$ Jean M. Elwing, MD, a and \\ Myron Gerson, $M^{d}$ \\ a Division of Pulmonary, Critical Care, and Sleep Medicine, University of Cincinnati College of \\ Medicine, Cincinnati, $\mathrm{OH}$ \\ b Division of Internal Medicine, University of Cincinnati College of Medicine, Cincinnati, OH \\ c Undergraduate Studies, University of Cincinnati College of Arts and Sciences, Cincinnati, OH \\ d Division of Cardiovascular Diseases, University of Cincinnati College of Medicine, Cincinnati, \\ $\mathrm{OH}$
}

Received Jan 24, 2021; accepted Mar 17, 2021

doi: 10.1007/s12350-021-02612-y

Background. The clinical significance of incidentally found RV abnormalities on low-risk SPECT studies is not well-defined. The objective of this study was to determine the predictive value of incidental right ventricular (RV) abnormalities identified on single photon emission computed tomography (SPECT) scans for mortality and pulmonary hypertension (PH).

Methods. We retrospectively analyzed all low-risk SPECT studies in patients without known coronary artery or pulmonary vascular disease, performed at our institution, from 2007-2020. Adjusted Cox proportional hazards models were used to evaluate the association between incidental RV abnormalities on low-risk SPECT studies and outcomes.

Results. Of the $\mathbf{4 7 6 1}$ patients included in the analysis, mortality events were present in 494, and echocardiographic PH was present in 619. Incidental RV abnormalities on low-risk SPECT studies were significantly and independently associated with all-cause mortality $(\mathrm{HR}=1.41, \mathrm{CI}$ $[1.07-1.86], P=0.0152)$ and echocardiographic PH (HR = 2.06, CI $[1.64-2.60], P<0.0001)$.

Conclusions. These data suggest incidental RV abnormalities found on low-risk SPECT imaging studies are significantly and independently associated with increased mortality and risk of developing echocardiographic $\mathrm{PH}$, and could identify high-risk patients for closer monitoring and additional diagnostic testing. (J Nucl Cardiol 2022;29:1903-14.)

Supplementary Information The online version of this article (h ttps://doi.org/10.1007/s12350-021-02612-y) contains supplementary material, which is available to authorized users.

The authors of this article have provided a PowerPoint file, available for download at SpringerLink, which summarises the contents of the paper and is free for re-use at meetings and presentations. Search for the article DOI on SpringerLink.com.

The authors have also provided an audio summary of the article, which is available to download as ESM, or to listen to via the JNC/ASNC Podcast.

Funding This work was supported by a Center for Clinical and Translational Science and Training Grant (2UL1TR001425-05A1).
Reprint requests: Arun Jose, MD, MS, Division of Pulmonary, Critical Care, and Sleep Medicine, University of Cincinnati College of Medicine, 6352-A, Medical Sciences Building, 231 Albert Sabin

Way, Cincinnati, OH 45267; josean@ucmail.uc.edu $1071-3581 / \$ 34.00$

Copyright (c) 2021 American Society of Nuclear Cardiology. 


\begin{tabular}{|ll|}
\hline Abbreviations & \\
EMERSE & Electronic Medical Record Search \\
& Engine \\
EMR & Electronic Medical Record \\
ICD-10 & International Classification of Diseases \\
& Codes 10th Edition \\
NDI & National Death Index \\
PH & Pulmonary Hypertension \\
RV & Right Ventricle \\
SPECT & Single Photon Emission Computed \\
& Tomography \\
TR & Tricuspid Regurgitant \\
\hline
\end{tabular}

See related editorial, pp. 1915-1918

\section{INTRODUCTION}

Single photon emission computed tomography (SPECT) imaging is a diagnostic tool to assess myocardial perfusion and risk of coronary artery disease, commonly used to identify patients presenting with symptoms of chest discomfort who may benefit from additional invasive diagnostic testing. ${ }^{1-4}$ Due to the anatomy of the cardiac chamber and the positioning of the camera system, the right ventricle (RV) is also visualized during SPECT studies, and information can be obtained regarding RV structure and function. ${ }^{5-9}$ In patients with pre-existing coronary artery disease or pulmonary vascular disease, the presence of RV abnormalities on SPECT imaging (including dilation and hypertrophy) have been associated with both disease severity and increased mortality, and may have prognostic significance. ${ }^{7,10-13}$ However, in patients without known coronary artery disease (as confirmed by low-risk or negative SPECT imaging studies), who do not have existing pulmonary vascular disease, the significance of incidentally observed RV abnormalities has not been defined. This scenario leads to a conundrum for the referring physician in determining the clinical implications of this finding, and what, if any, follow-up is needed for a patient with a low-risk study but evidence of RV abnormalities.

In order to address this knowledge gap and answer unresolved questions, we performed a large retrospective single center study to determine if there is an association between incidental RV abnormalities and mortality, hospital admission, and development of echocardiographic pulmonary hypertension $(\mathrm{PH})$ among a cohort of patients with low-risk SPECT studies. We hypothesized that incidental RV abnormalities would be significantly and independently predictive of these adverse clinical outcomes.

\section{METHODS}

\section{Data Sources}

We retrospectively analyzed all consecutive patients undergoing resting and/or rest/stress myocardial perfusion SPECT imaging studies at our center between January 7th 2007 and January 7th 2020. This date range was selected based on the earliest date (1/7/07) at which our EMR began to capture information on clinical imaging studies. All perfusion imaging was performed using either Tc-99 m-tetrofosmin or Tc-99 m-sestamibi radiotracers. Test protocols were approximately $87 \%$ one-day rest followed by stress, $5 \%$ two-day stress followed by rest, 5\% stress only, and 3\% rest only. Pharmacological stress was performed with intravenous dipyridamole $0.56 \mathrm{mg} / \mathrm{kg}$ over four minutes until 2009, and subsequently with regadenoson $0.4 \mathrm{mg}$ intravenously, with adjunctive exercise where possible. Patients unable to achieve $85 \%$ of maximum predicted heart rate with treadmill exercise were converted to pharmacological stress studies. Most imaging studies beginning in 2013 were performed on a cadmium-zinctelluride solid state camera system (D-SPECT, Spectrum Dynamics MEDICAL Inc) with routine acquisition in the upright and supine positions and no attenuation correction. Images were processed by iterative reconstruction using Spectrum Dynamics Cedar View and post-processed imaging reviewed on Corridor 4DM software using a dedicated normal file generated on the D-SPECT camera. Studies prior to 2013 were performed on a conventional SPECT camera and were processed with and without attenuation correction on Siemens Symbia Software, and reviewed on Corridor 4DM software (Invia Medical Imaging Solutions, Ann Arbor, Michigan). The majority of studies were interpreted by one of two nuclear cardiology board-certified physicians. There was excellent consistency between readers, with a random sample of 16 studies re-read blinded by each reviewer demonstrating $93.8 \%$ agreement (15 of 16) on identifying the RV as abnormal, $86.7 \%$ agreement on identifying RV dilation (13 of 15), and 100\% agreement on identifying RV hypertrophy (9 of 9). SPECT studies were identified by EPIC procedure codes (IMG417, IMG418, IMG1964, and IMG6075), and procedure reports were interrogated using the Electronic Medical Record Search Engine (EMERSE) text mining tool. ${ }^{14}$ This study was found to be exempt from informed consent, reviewed, and approved by our Institutional Review Board (IRB 2019-1282). 


\section{Study Population}

All SPECT studies that were negative for evidence of myocardial ischemia and classified as low-risk, based on our institution's standard SPECT reporting template for risk classification (low, medium/moderate, or high), validated using EMERSE to identify the absence of key phrases ("abnormal study", "critical finding", "moderate cardiac risk', "medium cardiac risk', "high cardiac risk", “elevated cardiac risk', “decreased perfusion") and the presence of key phrases ("normal left ventricular perfusion", "normal myocardial perfusion',), were included in the final analysis. By definition, these criteria excluded any studies with reversible defects, and studies with transient stress dilatation, from the final analysis. For subjects with multiple SPECT studies performed, only the first set of tests was included in the final analysis. Subjects with cardiac or pulmonary conditions present prior to the date of initial SPECT study that could affect the presence of RV abnormalities on SPECT were excluded. ${ }^{10}$ These conditions, identified using the 10th iteration of the International Classification of Diseases Codes (ICD-10), were heart failure (ICD-10 I50), pulmonary hypertension (ICD-10 I27), previous myocardial infarction (ICD-10 I20-I25), and valvular heart disease (ICD-10 I34-I37). Duplicate medical records were excluded. This study was reviewed and approved by our Institutional Review Board (IRB 2019-1282).

\section{Exposure}

The main exposure was the presence or absence abnormalities of the RV on SPECT imaging (Figure 1). Our institution's SPECT reporting template requires assessment of $\mathrm{RV}$ as normal or abnormal. Abnormal RV findings are then routinely categorized as dilation and/or hypertrophy. Right ventricular size is defined as large if the RV appears visually larger than the left ventricle. Right ventricular hypertrophy was defined as uptake intensity of $50 \%$ or greater compared to the uptake intensity of the left ventricle, with an RV thickness visually comparable to the thickness of the left ventricle. ${ }^{8,9}$ Using EMERSE, studies were classified as either positive or negative for abnormalities of the right ventricle, based on containing one of the following key phrases: "right ventricular hypertrophy", "right ventricular dilation", or "increased right ventricular uptake", Low risk SPECT studies reported normal left ventricular perfusion, had no evidence of myocardial ischemia, and did not have any of those phrases regarding the right ventricle in the study report.

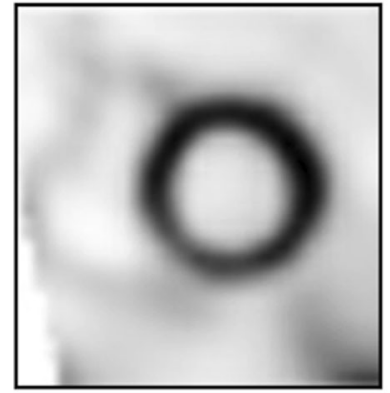

RV Dilation

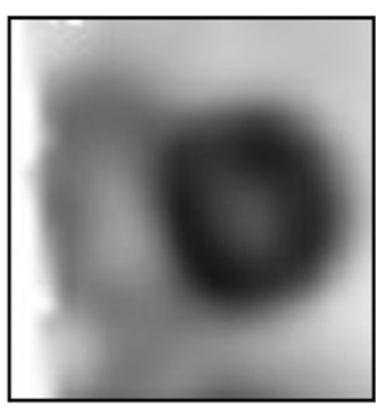

RV Hypertrophy
Figure 1. Representative Images of Right Ventricular Dilation and Hypertrophy. Representative images of right ventricular dilation (left) and hypertrophy (right). Right ventricular dilation demonstrates enlargement of the right ventricle with loss of the normal semilunar shape. Right ventricular hypertrophy demonstrates wall thickening with increased right ventricular and septal uptake, as well as septal flattening.

\section{Covariates}

Demographic information at the time of SPECT study (age, gender, race, and body mass index (BMI) was obtained from the EMR. Racial categories included White, Black, Asian, Hispanic, American Indian/Alaskan, or Other. Using ICD-10 codes, information on potentially confounding comorbid diagnoses associated with both mortality and either cardiac chamber hypertrophy or dilation at the time of the initial SPECT study was also obtained. These comorbidities including Diabetes Mellitus (DM, ICD-10 E8-E13) or Kidney Disease (CKD, ICD-10 N17-N19), Hypertension (HTN, ICD-10 I10-I16), and Sleep Apnea (OSA, ICD-10 G47.3) and Chronic Obstructive Pulmonary Disease (COPD, ICD10 J40-J44. ${ }^{15-19}$

\section{Outcome}

The primary outcome of this study was time to allcause mortality, obtained from review of the EMR. Time zero was the date of the index SPECT test, and patients were followed until either an event occurred or the study period ended (February $1^{\text {st }}, 2020$ ). Vital status, hospitalizations, and echocardiographic testing outside of the study institution were routinely assessed from each clinical encounter using documentation in the patient chart.

Secondary outcomes included time to all-cause hospital admission and time to an echocardiographic diagnosis of PH following initial SPECT study. Admission was defined as a hospital encounter which included an admission history and physical, a discharge summary, 
and was billed as an admission (as compared to hospital observation) in the EMR. PH on echocardiogram was defined as a tricuspid regurgitant (TR) jet velocity of greater than 2.8 meters per second, as based on the cutoff used to identify intermediate or high echocardiographic probability of $\mathrm{PH}$ from the most recent guidelines for the screening and diagnosis of pulmonary hypertension. ${ }^{20,21}$ All $\mathrm{PH}$ patients were required to have normal pulmonary pressures by echocardiography at the time of SPECT imaging, and a diagnosis of echocardiographic $\mathrm{PH}$ was confirmed by the progression of echocardiographic pulmonary pressures and an increase in the TR jet velocity to above 2.8 meters per second over the course of the study. Patients without PH were required to demonstrate a normal $\mathrm{TR}$ jet velocity $\leq 2.8$ $\mathrm{m} / \mathrm{s}$ (with normal estimated echocardiographic pulmonary pressures) at all echocardiographic studies following the initial SPECT study. Patients with a preexisting diagnosis of $\mathrm{PH}$, or those without baseline echocardiographic testing prior to SPECT imaging to rule-out pre-existing $\mathrm{PH}$, were excluded from the study. All diagnoses of $\mathrm{PH}$ were reviewed and validated by a pulmonary vascular disease specialist (A Jose).

\section{Statistical Analyses}

Differences in demographic and clinical characteristics between groups were compared using the MannWhitney-Wilcoxon test for continuous variables and Fisher's Exact test for categorical variables, respectively. Time to event data for the outcome measures of all-cause mortality, all-cause hospitalization, and echocardiographic diagnosis of $\mathrm{PH}$, were modeled using a Cox proportional hazards model. ${ }^{22}$ All univariable models were adjusted for demographics (age, gender, race), BMI, and comorbidities (DM, HTN, CKD, OSA, COPD). The proportional hazards assumption was examined for all models using scaled Schoenfeld residuals. A two-tailed $P$-value $\leq 0.05$ was significant. Missing data were subject to pairwise deletion. Analyses were performed using $\mathrm{R}$ version 3.6.1 (R Foundation for Statistical Computing, Vienna, Austria).

\section{RESULTS}

\section{Study Population Characteristics}

A total of 12,883 unique SPECT imaging reports were identified by our EMR between 1/7/2007 and 1/7/ 2020. Of these, 4761 met study criteria and were included in the final analysis (Figure 2). The majority of SPECT studies were ordered on patients in the emergency department setting $(3241,68.1 \%)$. A total of 36 patients were excluded from the final analysis due to

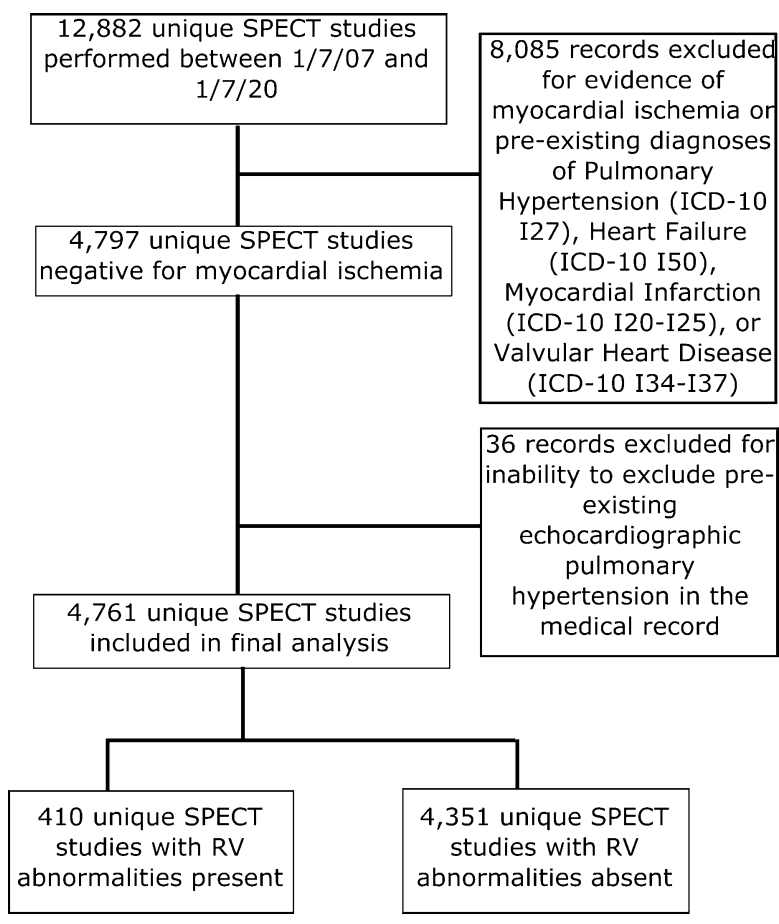

Figure 2. Flow of Patients Through the Study. Flow of patients through the study and determination of final groups for analysis. $R V$, right ventricle; SPECT, single photon emission computed tomography; $I C D$, International Classification of Diseases coding.

developing echocardiographic $\mathrm{PH}$ over the course of the study, but no normal echocardiogram without $\mathrm{PH}$ at the time of SPECT imaging to confirm they did not have pre-existing $\mathrm{PH}$. The cohort was evenly split between males $(50.8 \%)$ and females $(49.2 \%)$, with a median age of 50 years old and a median BMI of $30.3 \mathrm{~kg} / \mathrm{m}^{2}$ (Table 1). The median time of follow-up until death or censoring was 95.5 months. RV abnormalities were present in 410 subjects $(8.6 \%)$, with the majority (288, $70.2 \%$ ) exhibiting RV hypertrophy. Those with RV abnormalities were significantly more likely to be older, male, and significantly less likely to have comorbid HTN. Mortality events were present in 494 subjects $(10.4 \%)$. A slight majority $(57.3 \%)$ of our cohort had complete vital status documented at the time of censoring. Patients with RV abnormalities were significantly more likely to have a mortality event as compared to those without RV abnormalities on SPECT imaging (18.5\% versus $9.6 \%, P<0.0001)$, and develop echocardiographic PH (25.9\% versus $11.8 \%, P<0.0001)$, but there was no significant difference in hospital admission following initial SPECT study between the groups (56.6\% versus $51.8 \%, P=0.0702$ ).

A total of 2142 patients $(45 \%)$ did not have echocardiographic testing following initial SPECT imaging to evaluate for echocardiographic $\mathrm{PH}$, and 
Table 1. Baseline characteristics of study cohort

\begin{tabular}{|c|c|c|c|c|}
\hline Clinical variable & $\begin{array}{c}\text { Full cohort } \\
(N=4761) \\
\text { Median (IQR) } \\
\text { or frequency } \\
(\%)\end{array}$ & $\begin{array}{c}\text { RV abnormality } \\
\text { present } \\
(\mathbf{N}=410) \\
\text { Median (IQR) or } \\
\text { frequency (\%) }\end{array}$ & $\begin{array}{c}\text { RV abnormality } \\
\text { absent } \\
\text { (N=4351) } \\
\text { Median (IQR) or } \\
\text { frequency (\%) }\end{array}$ & $P$ value \\
\hline Age (years) & $50(43-58)$ & $53(47-59)$ & $50(43-58)$ & $<0.0001$ \\
\hline Female gender & $2345(49.2 \%)$ & $84(20.5 \%)$ & $2261(51.9 \%$ & $<0.0001$ \\
\hline Race & & & & 0.5259 \\
\hline White & $2371(49.8 \%)$ & $216(52.7 \%)$ & $2155(49.5 \%)$ & \\
\hline Black & $2113(44.4 \%)$ & $169(41.2 \%)$ & $1944(44.7 \%)$ & \\
\hline Asian & $47(1 \%)$ & $5(1.2 \%)$ & $42(0.9 \%)$ & \\
\hline Other & $230(4.8 \%)$ & $20(4.9 \%)$ & $210(4.8 \%)$ & \\
\hline BMI $\left(\mathrm{kg} / \mathrm{m}^{2}\right)$ & $30.3(26.0-35.5)$ & $30.1(25.8-35.0)$ & $30.3(26.0-35.5)$ & 0.9204 \\
\hline HTN & $277(5.8 \%)$ & $8(2.0 \%)$ & $269(6.2 \%)$ & 0.0001 \\
\hline $\mathrm{DM}$ or $\mathrm{CKD}$ & $11(0.2 \%)$ & $0(0 \%)$ & $11(0.3 \%)$ & 0.6164 \\
\hline COPD or OSA & $22(0.5 \%)$ & $0(0 \%)$ & $22(0.5 \%)$ & 0.2524 \\
\hline Follow-up Time (months) & $95.5(40.4-130.7)$ & $90.5(33.1-132.7)$ & $95.8(41.7-130.5)$ & 0.2771 \\
\hline Mortality & $494(10.4 \%)$ & $76(18.5 \%)$ & $418(9.6 \%)$ & $<0.0001$ \\
\hline Time to mortality (months) & $69.8(36.0-101.8)$ & $73.4(25.2-106.2)$ & $69.4(38.6-100.7)$ & 0.4480 \\
\hline Admission & $2487(52.2 \%)$ & $232(56.6 \%)$ & $2255(51.8 \%)$ & 0.0702 \\
\hline Time to admission (months) & $27.7(8.1-60.9)$ & $23.8(6.0-60.1)$ & $28.0(8.5-60.9)$ & 0.1435 \\
\hline PH on echocardiogram & $619(13.0 \%)$ & $106(25.9 \%)$ & $513(11.8 \%)$ & $<0.0001$ \\
\hline $\begin{array}{l}\text { TR Jet velocity for those with } \\
\text { echocardiographic PH (m/ } \\
\text { s) }\end{array}$ & $3.08(2.96-3.35)$ & $3.08(2.92-3.46)$ & $3.08(2.95-3.35)$ & 0.7895 \\
\hline $\begin{array}{l}\text { Time to PH diagnosis } \\
\text { (months) }\end{array}$ & $37.6(5.0-84.0)$ & $20.0(0.08-77.8)$ & $41.8(7.3-84.1)$ & 0.0106 \\
\hline RV hypertrophy & - & $288(70.2 \%)$ & - & \\
\hline RV dilation & - & $146(35.6 \%)$ & - & \\
\hline $\begin{array}{l}\text { Mean pulmonary arterial } \\
\text { pressure on } \mathrm{RHC}(\mathrm{mmHg})\end{array}$ & - & $32(28-42)$ & - & \\
\hline $\begin{array}{l}\text { Pulmonary capillary wedge } \\
\text { pressure on } \mathrm{RHC}(\mathrm{mmHg})\end{array}$ & - & $20^{13-25}$ & - & \\
\hline $\begin{array}{l}\text { Pulmonary vascular } \\
\text { resistance on RHC } \\
\text { (Woods Units) }\end{array}$ & - & $2.98(1.73-5.04)$ & - & \\
\hline
\end{tabular}

Baseline characteristics of study cohort, separated into those with incidental RV abnormalities and those without. Right-hand column is Mann-Whitney-Wilcoxon or Fisher's Exact Test $P$-value, for continuous or categorical data, respectively.

$R V$, Right ventricle; $B M I$, Body Mass Index; HTN, hypertension; DM, Diabetes Mellitus; CKD, chronic kidney disease, OSA, Obstructive Sleep Apnea; COPD, Chronic Obstructive Pulmonary Disease; $P H$, pulmonary hypertension; TR Tricuspid Regurgitant; RHC, Right Heart Catheterization

were not included in the $\mathrm{PH}$ secondary endpoint analysis. Among those with echocardiographic PH, a total of $81(19.6 \%)$ had additional right heart catheterization testing data available. Within these patients with echocardiographic $\mathrm{PH}$ who underwent invasive hemodynamics testing, the majority $(\mathrm{N}=75,92.5 \%)$ had pulmonary hypertension confirmed hemodynamically, with a median mean pulmonary arterial pressure of $32 \mathrm{mmHg}$. PH due to left heart disease, as evidenced by a pulmonary capillary wedge pressure of $>15 \mathrm{mmHg}$, was the most common etiology of PH, occurring in 48 of the patients $(64 \%)$ with catheterization-confirmed $\mathrm{PH}$. 


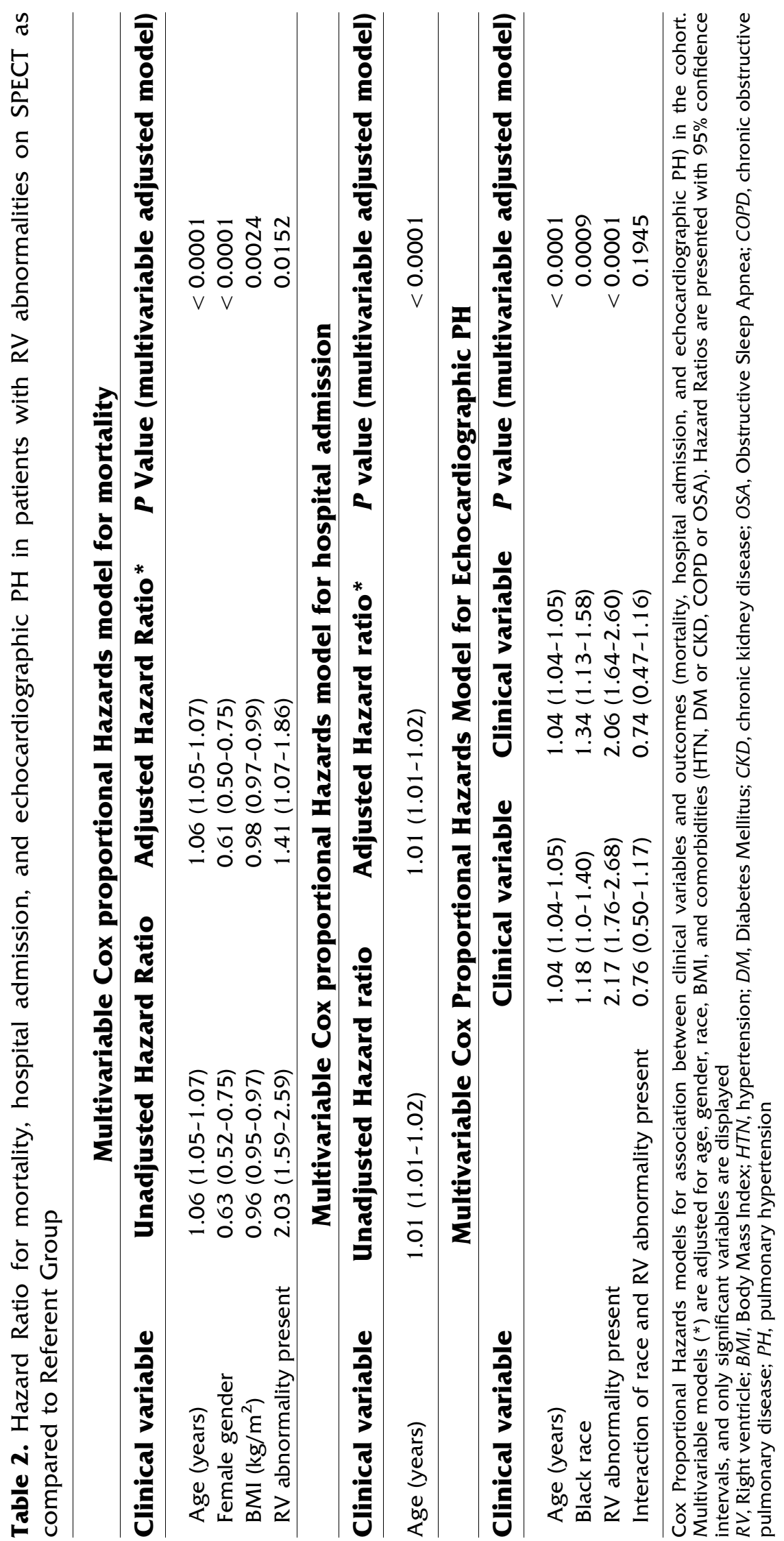


Time to Event Plot for Unadjusted Mortality Stratified by RV Abnormalities on SPECT
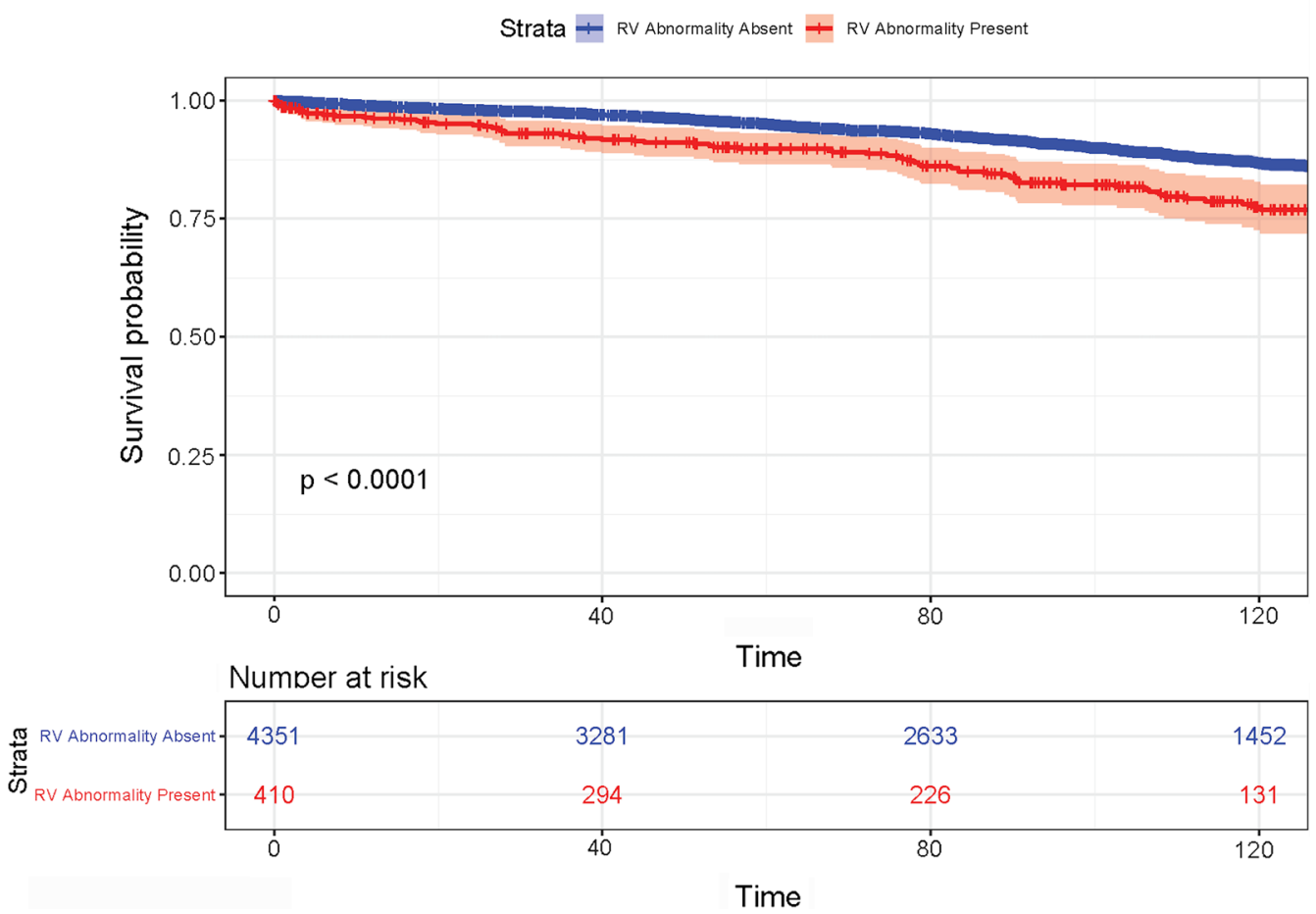

Figure 3. Time to Event Plot for Unadjusted Mortality Stratified by RV Abnormalities on SPECT. Time to event plot for unadjusted mortality stratified for presence or absence of RV abnormalities on SPECT imaging. Log-rank test $P<0.0001$. Time is displayed in months. $R V$, Right ventricle; $S P E C T$, single photon emission computed tomography.

\section{Association of RV Abnormalities on SPECT with Adverse Outcomes}

Compared with the reference group, those with RV abnormalities present on SPECT imaging had a significantly higher hazard ratio mortality (Hazard Ratio 1.41, 95\% Confidence Interval 1.07-1.86) (Table 2, Figure 3). This association between RV abnormalities on SPECT imaging and adverse outcomes persisted even after adjustment for age, gender, race, BMI, and clinical comorbidities (HTN, CKD, DM, COPD, and OSA). In the adjusted model, higher age was also associated with increased mortality (Hazard Ratio 1.06, $P<0.0001$ ), and both female gender (Hazard Ratio 0.61, $P<0.0001$ ) and BMI (Hazard Ratio 0.98, $P=0.0024)$ were protective. After stratification of BMI by quartiles with comparison to a reference quartile (reference quartile median BMI $29.2 \mathrm{~kg} / \mathrm{m}^{2}$ ) and multivariable adjustment, a significant association with mortality was observed for both the lowest (Hazard Ratio 1.47, $P=0.001$ ) and highest (Hazard Ratio 1.91, $P<0.0001$ ) BMI quartiles (Table 3 ).
RV abnormalities on SPECT imaging were also significantly associated with development of echocardiographic PH (Hazard Ratio 2.06, 95\% Confidence Interval 1.64-2.60). Increased age (Hazard Ratio 1.04, $P<0.0001$ ) and Black race (Hazard Ratio 1.34, $P=0.0009)$ were also significantly associated with the development of echocardiographic $\mathrm{PH}$ in the adjusted model. There was no significant association between RV abnormalities on SPECT imaging and hospital admission, and only increased age was significantly associated with admission in the adjusted model (Hazard Ratio 1.01, 95\% Confidence Interval 1.01-1.02).

RV hypertrophy was both independently and significantly associated with both mortality and development of echocardiographic $\mathrm{PH}$, even after adjustment for demographics and comorbid conditions (Table 4). RV dilation was only associated with risk of developing echocardiographic PH after adjustment. Among those with RV abnormalities present on SPECT imaging, there was no association between the individual abnormalities present (RV dilation or hypertrophy) 
Table 3. Hazard Ratio for Mortality in Patients with RV Abnormalities on SPECT Stratified by BMI Quartiles

Multivariable Cox Proportional Hazards Model for Mortality

\begin{tabular}{|c|c|c|c|}
\hline Clinical variable & $\begin{array}{c}\text { BMI }(\mathbf{k g} / \\
\left.\mathbf{m}^{2}\right)\end{array}$ & $\begin{array}{c}\text { Adjusted Hazard } \\
\text { ratio* }\end{array}$ & $\begin{array}{c}P \text { value (multivariable adjusted } \\
\text { model) }\end{array}$ \\
\hline Age (years) & & $1.06(1.06-1.07)$ & $<0.0001$ \\
\hline Female gender & & $0.56(0.46-0.67)$ & $<0.0001$ \\
\hline $\begin{array}{l}\text { RV abnormality } \\
\text { present }\end{array}$ & & $1.50(1.67-1.93)$ & 0.0016 \\
\hline \multicolumn{4}{|l|}{ BMI quartile } \\
\hline Reference & $\begin{array}{l}29.2(28.0- \\
30.5)\end{array}$ & - & - \\
\hline Low & $\begin{array}{l}23.9(21.7- \\
25.5)\end{array}$ & $1.47(1.17-1.85)$ & 0.001 \\
\hline High & $\begin{array}{l}34.9(33.2- \\
37.2)\end{array}$ & $0.99(0.78-1.29)$ & 0.933 \\
\hline Very High & $\begin{array}{l}45.0(42.3- \\
49.3)\end{array}$ & $1.91(1.45-2.52)$ & $<0.0001$ \\
\hline
\end{tabular}

Cox proportional hazards models for association between clinical variables and outcome of mortality. Multivariable models ( ${ }^{*}$ ) are adjusted for age, gender, race, and comorbidities (HTN, DM or CKD, COPD or OSA). BMI is grouped into quartiles, with median BMI of 29.2 as reference. Hazard Ratios are presented with $95 \%$ confidence intervals, and only significant variables are displayed

$R V$, Right ventricle; $B M I$, Body Mass Index; HTN, hypertension; DM, Diabetes Mellitus; CKD, chronic kidney disease; OSA, Obstructive Sleep Apnea; COPD, Chronic Obstructive Pulmonary Disease

Table 4. Hazard ratio for mortality and echocardiographic PH stratified by RV abnormality

\begin{tabular}{|c|c|c|c|}
\hline Clinical variable & Hazard Ratio* & 95\% Confidence interval & $P$ value \\
\hline \multicolumn{4}{|c|}{ Multivariable Cox Proportional Hazards Models for Mortality } \\
\hline \multicolumn{4}{|c|}{ Full cohort of patients $(\mathrm{N}=4761)$} \\
\hline RV hypertrophy & 1.49 & $1.10-2.03$ & 0.011 \\
\hline RV dilation & 1.01 & $0.62-1.63$ & 0.977 \\
\hline \multicolumn{4}{|c|}{ RV abnormality present $(\mathrm{N}=410)$} \\
\hline RV hypertrophy & 1.10 & $0.65-1.84$ & 0.733 \\
\hline RV dilation & 1.00 & $0.62-1.62$ & 0.992 \\
\hline \multicolumn{4}{|c|}{ Multivariable Cox proportional Hazards models for echocardiographic PH } \\
\hline \multicolumn{4}{|c|}{ Full cohort of patients $(\mathrm{N}=4761)$} \\
\hline RV hypertrophy & 1.90 & $1.46-2.47$ & $<0.001$ \\
\hline RV dilation & 1.59 & $1.09-2.32$ & 0.016 \\
\hline \multicolumn{4}{|c|}{$\mathrm{RV}$ abnormality present $(\mathrm{N}=410)$} \\
\hline RV hypertrophy & 0.90 & $0.59-1.38$ & 0.641 \\
\hline RV dilation & 1.07 & $0.72-1.60$ & 0.745 \\
\hline
\end{tabular}

Cox proportional hazards model for association between clinical variables and mortality as an outcome. Multivariable models (*) in full cohort are adjusted for age, gender, race, BMI, and comorbidities (HTN, DM or CKD, COPD or OSA)

$R V$, Right ventricle; $P H$, pulmonary hypertension 
and either mortality or development of echocardiographic $\mathrm{PH}$ in our cohort.

\section{DISCUSSION}

This analysis demonstrates an association between incidental RV abnormalities on SPECT imaging and an increased risk of both all-cause mortality and the development of echocardiographic PH. This association persisted even after adjustment for demographics and potentially confounding clinical comorbidities. To our knowledge this is the first reported association between incidental RV abnormalities on SPECT imaging and clinical outcomes in patients without known cardiopulmonary disease, and identifies a potential role for SPECT imaging as a risk stratification tool and screening modality for pulmonary vascular disease in these patients. Furthermore, the findings support the routine reporting of $\mathrm{RV}$ findings on myocardial perfusion SPECT imaging.

In our adjusted Cox proportional hazards model, we observed mortality was significantly associated with incidental RV abnormalities on SPECT imaging in patients without known coronary artery or pulmonary vascular disease. Although our study was not designed to evaluate mechanisms, we hypothesize this association may partly reflect underlying sub-clinical or undiagnosed pulmonary vascular disease in these patients, as patients with incidental RV abnormalities also demonstrated a significant association with risk of echocardiographic PH. The link between incidental RV abnormalities and echocardiographic PH does not fully explain the mortality association we observed, as incidental RV abnormalities were an independent mortality risk factor ever after adjustment for degree of echocardiographic PH (as measured by the TR jet velocity) (Table S1). Abnormalities of the RV and pulmonary vasculature have previously been shown to predict mortality, adverse clinical outcomes, and risk of echocardiographic $\mathrm{PH}$ in patients with underlying coronary artery disease undergoing SPECT imaging. The results of our work extend this prognostic value to patients with low-risk SPECT studies. ${ }^{5,10,12,23}$ Additionally, our work identifies incidental RV abnormalities on SPECT imaging as a potential marker predicting development of pulmonary vascular disease. Although this association between $\mathrm{PH}$ and $\mathrm{RV}$ abnormalities on SPECT has been observed previously in the literature, our study is the first to link the risk of PH with mortality as a clinical outcome in patients at low risk for coronary artery disease, and suggests a role for SPECT imaging in identifying at-risk patients who might benefit from additional diagnostic evaluation or closer clinical monitoring. ${ }^{7,13,24-26}$ Given the frequent and widespread use of SPECT imaging to risk-stratify patients for coronary artery disease, the use of SPECT imaging findings in this fashion could potentially result in earlier diagnosis of pulmonary vascular disease and improved clinical outcomes in a significant number of patients. ${ }^{27,28}$

In our study we also observed a significant association with Black race and risk of echocardiographic $\mathrm{PH}$ among patients with low-risk SPECT studies. However, after evaluating our model for the interaction between race and incidental RV abnormalities on SPECT imaging, no significant relationship was observed (Table 1). Consequently, we are unable to draw further conclusions regarding the relationship between race, incidental RV abnormalities on SPECT imaging, and risk of echocardiographic PH.

Unexpectedly we observed a protective effect between BMI and mortality in this cohort, and although this effect was quite weak, it remained a significant effect in our multivariable model even after adjustment. This may be reflective of the so-called "obesity paradox" observed in epidemiologic disease studies, where the paradoxically protective effect of an elevated BMI results from an underlying mortality effect of extremely low and high BMI measures. ${ }^{29,30}$ Indeed, after stratifying our cohort by BMI quartiles, and after adjustment, we found a significantly elevated risk of mortality in both the lowest and highest BMI quartiles, with the greatest hazard in the most obese patients, supporting an association between mortality and extremes of BMI in this cohort (Table 3).

We did not observe any association between incidental RV abnormalities on SPECT imaging and hospital admissions in our cohort, and the only significant predictor of admissions was age. This finding was unexpected in light of the increased mortality seen in those with incidental RV abnormalities on SPECT imaging, and may be a result of the broad criteria used to define hospital admission, with no distinctions made for the diagnosis requiring hospitalization. As a result, a cardiopulmonary diagnosis for admission (such as newonset heart failure) and a presumably unrelated diagnosis (such as psychiatric illness) would be indistinguishable, as would an admission to a medical floor versus an intensive care unit admission, and both would qualify as a hospital admission event. We believe this source of confounding may have limited our ability to definitively associate incidental RV abnormalities on SPECT imaging with risk of hospitalization.

Among patients with abnormal RV imaging studies, RV hypertrophy was more strongly associated with outcomes (mortality, echocardiographic PH development) in our cohort as compared with RV dilation. This was unexpected, as in pulmonary vascular disease patients hypertrophy of the $\mathrm{RV}$ is more commonly 
accepted to be an adaptive response, and dilation a maladaptive response, and we would have expected RV dilation to show the stronger association. ${ }^{21}$ However, given the higher number of patients who exhibited RV hypertrophy in our cohort, and the reliance upon subjective size criteria for RV dilation in our institutional SPECT template, it is possible this stronger association is secondary to selection bias, and we would hesitate to draw definitive conclusions regarding the relative prognostic value of individual RV abnormalities on SPECT imaging with clinical outcomes. Additionally, in our study we did not distinguish between abnormalities of the RV observed at stress versus those at rest, but it is well established, particularly for pulmonary vascular disease, that physiologic stress during cardiopulmonary testing often provides unique prognostic information as compared to testing at rest. $^{31,32}$ Further investigation into specific patterns of $\mathrm{RV}$ dysfunction, the potential difference in predictive value between resting and stress abnormalities, and the reliability of subjective SPECT findings such as RV dilation are warranted, to better understand the prognostic information offered by incidental RV abnormalities identified on SPECT imaging.

Our study has several strengths, including combining electronic medical record results with individual chart review of all patients to verify outcomes (mortality, echocardiographic $\mathrm{PH}$, hospital admission), a large and robust dataset, a standardized SPECT imaging report template that included cardiac risk (low, medium, or high) and required assessment of the RV and avoidance of confounding signals for morbidity and mortality by censoring our data prior to the emergence of the SARS-CoV-2 pandemic in the United States. ${ }^{33}$

There are also a number of limitations that merit discussion. This was a retrospective single center study focused on patients with low-risk or negative SPECT imaging studies, primarily 1-day D-SPECT studies, the results should not be generalized to other patient populations, imaging systems, or imaging protocols, and will require further validation in a larger multicenter prospective fashion. Although the majority of SPECT studies in our cohort were 1-day rest-stress studies, we cannot exclude a difference in physiologic effect of both the presence and type of stress on the right ventricle, and further investigation on the interaction between stress and the right ventricle is needed to clarify this relationship.

We relied upon ICD-10 coding to identify comorbid medical conditions, which are known to be limited tools to reflect clinical status, and may have under-reported the true incidence of these conditions in our study. ${ }^{34}$
Additionally, comorbid medical conditions were only captured at the time of initial SPECT imaging, and would not have identified patients who carried undiagnosed medical comorbidities or later developed these conditions. These two limitations could affect the relationship between RV abnormalities on SPECT imaging and clinic outcomes in our adjusted multivariable models.

The majority of SPECT imaging studies in our cohort were ordered on patients in the emergency department setting, and we observed a higher than expected mortality rate in our cohort of $9.6 \%$ over an observation period of approximately 8 years. We suspect this may reflect the overall poor access to care experienced by these patients, as reliance on the emergency department for medical care (such as workup of chest discomfort with SPECT imaging studies) is known to be associated with increased mortality and adverse outcomes. ${ }^{35,36}$ Our study was not designed to study this association, and we are unable to draw further conclusions on this potential relationship between imaging study location and mortality rate, but the higher than expected mortality rate further highlights the potential benefit provided by early identification of patients at high risk for mortality using incidental RV abnormalities identified on SPECT imaging.

Although all patient records were individually examined for mortality and hospital admission outcomes, we only had access to those EMR records within our Medical Center and associated hospitals, and were unable to supplement this information with federal mortality data, such as the National Death Index (NDI), to more fully capture all mortality events. ${ }^{37}$ This could have led to an under-estimation of the true mortality rate, which could have affected our conclusions. Although the NDI would capture all mortality events and is considered the "gold standard" for vital status capture, it is only updated annually without full records beyond 2018, and EMR records alone are still effective at capturing the majority of mortality events. ${ }^{38}$ Consequently, as a minority of patients $(2204,46.3 \%)$ in our cohort did not have full vital status information at the time of censoring, including only 1621 (34\%) patients censored before 12/31/18, and the patients with incomplete vital status information had comparable clinical and demographic characteristics to our full cohort (Table S2), we do not anticipate this limitation to significantly alter the conclusions of our work.

We chose to exclude patients who developed echocardiographic $\mathrm{PH}$, but did not have an earlier echocardiogram without PH by TR jet criteria, to avoid capturing prevalent $\mathrm{PH}$ patients diagnosed at our Center 
or another institution (Figure 2). Of the 36 patients excluded, ten $(28 \%)$ had evidence of incidental RV abnormalities on SPECT imaging. Excluding these patients, which would constitute less than $1 \%$ of our total dataset, may have removed some new diagnoses of echocardiographic PH from the final analysis, and although unlikely it is possible this could have affected our results.

We selected a secondary outcome of echocardiographic $\mathrm{PH}$, based on the TR jet velocity, but not all echocardiographic studies are able to accurately capture the tricuspid valve and measure the TR jet, and not all patients with SPECT testing underwent a follow-up echocardiogram, introducing some selection bias to this outcome. We attempted to mitigate this limitation by defining echocardiographic $\mathrm{PH}$ in a similar fashion to the most recent guidelines for the screening and diagnosis of pulmonary hypertension. ${ }^{20,21}$ It should be noted that, although right heart catheterization is required to confirm $\mathrm{PH}$, the presence of echocardiographic $\mathrm{PH}$ is itself associated with mortality, making this a clinically important outcome to study. ${ }^{39-41}$ Additionally, in the 81 patients who had both echocardiography and right heart catheterization in our cohort, $\mathrm{PH}$ was present in the vast majority (75 patients or $92.5 \%$ ), with a median mean pulmonary arterial pressure of $32 \mathrm{mmHg}$ (interquartile range $27-42 \mathrm{mmHg}$ ), and post-capillary hemodynamics with a pulmonary capillary wedge pressure more than $15 \mathrm{mmhg}$ present in 48 patients $(65 \%)$, confirming the clinical significance of this outcome in our study.

In summary, we demonstrate that incidental RV abnormalities found on low-risk SPECT imaging studies are significantly and independently associated with both all-cause mortality and risk of developing echocardiographic pulmonary hypertension. This finding may identify patients at higher risk of adverse clinical outcomes, and supports future investigation into the role of incidental RV abnormalities on SPECT imaging studies as a screening tool for pulmonary vascular disease and a marker of increased mortality risk in other patient populations.

\section{NEW KNOWLEDGE GAINED}

Imaging cardiovascular biomarkers with diagnostic and prognostic value are useful in guiding the clinical management of patients undergoing SPECT imaging studies. In this study, we identify incidental RV abnormalities on low-risk SPECT imaging studies as significantly and independently associated with patient mortality and risk of developing echocardiographic $\mathrm{PH}$, and describe how this finding can help identify high-risk patients who may benefit from additional screening and clinical follow-up.

\section{Disclosures}

No conflicts of interest exist relevant to this work for Arun Jose, Christine Zhou, Rachel Baker, Jackson Walker, Nicholas Kurek Robert E. O'Donnell, Jean M. Elwing, Myron Gerson.

\section{References}

1. Taban Sadeghi M, Mahmoudian B, Ghaffari S, Moharamzadeh P, Ala A, Pourafkari L, et al. Value of early rest myocardial perfusion imaging with SPECT in patients with chest pain and non-diagnostic ECG in emergency department. Int J Cardiovasc Imaging 2019;35(5):965-71.

2. Fordyce CB, Newby DE, Douglas PS. Diagnostic strategies for the evaluation of chest pain: clinical implications from SCOTHEART and PROMISE. J Am Coll Cardiol 2016;67(7):843-52.

3. Loong CY, Anagnostopoulos C. Diagnosis of coronary artery disease by radionuclide myocardial perfusion imaging. Heart 2004;90(5S):v2-9.

4. Klocke FJ, Baird MG, Lorell BH, Bateman TM, Messer JV, Berman DS, et al. ACC/AHA/ASNC guidelines for the clinical use of cardiac radionuclide imaging-executive summary: a report of the American College of Cardiology/American Heart Association Task Force on Practice Guidelines (ACC/AHA/ASNC Committee to Revise the 1995 Guidelines for the Clinical Use of Cardiac Radionuclide Imaging). J Am Coll Cardiol 2003;42(7):1318-33.

5. Movahed M. Gated SPECT can detect abnormal RV structure or function. Nat Rev Cardiol 2011;8:290.

6. Aepfelbacher FC, Yeon SB, Ho KKL, Parker JA, Danias PG. ECG-gated $99 \mathrm{mTc}$ single-photon emission CT for assessment of right ventricular structure and function: is the information provided similar to echocardiography? Chest 2003;124(1):227-32.

7. Ohira H, Beanlands RS, Davies RA, Mielniczuk L. The role of nuclear imaging in pulmonary hypertension. J Nucl Cardiol 2015;22(1):141-57.

8. Farag AA, Heo J, Tauxe L, Dhambhvani P, Germano G, et al. Detection and quantitation of right ventricular reversible perfusion defects by stress SPECT myocardial perfusion imaging: a proofof-principle study. J Nucl Cardiol 2019;26:266-71.

9. Dorbala S, Anathasubramaniam K, Armstrong IS, Chareonthaitawee P, DePuey EG, Einstein AJ, et al. Single photon emission computed tomography (SPECT) myocardial perfusion imaging guidelines: instrumentation, acquisition, processing, and interpretation. J Nucl Cardiol 2018;25:1784-846.

10. Mannting F, Zabrodina YV, Dass C. Significance of increased right ventricular uptake on $99 \mathrm{mTc}$-sestamibi SPECT in patients with coronary artery disease. J Nucl Med 1999;40(6):889-94.

11. Williams KA, Schneider CM. Increased stress right ventricular activity on dual isotope perfusion SPECT: a sign of multivessel and/or left main coronary artery disease. J Am Coll Cardiol 1999;34(2):420-7.

12. Ko K, Wu Y, Liu C, Cheng M, Yen R, Tzen K. Clinical significance of right ventricular activity on treadmill thallium-201 myocardial single-photon emission computerized tomography using cadmium-zinc-telluride cameras. Nucl Med Commun 2016;37(6):650-6.

13. Murarka S, Movahed MR. Review of Movahed's sign (D shaped left ventricle seen on gated SPECT) suggestive of right ventricular overload. Int J Cardiovasc Imaging 2010;26(5):553-7.

14. Hanauer DA, Barnholtz-Sloan JS, Beno MF, Fiol GD, Durbin EB, Gologorskaya $\mathrm{O}$, et al. Electronic medical record search engine (EMERSE): an information retrieval tool for supporting cancer research. JCO Clin Cancer Inform 2020;4:454-63. 
15. Eguchi K, Boden-Albala B, Jin Z, Rundek T, Sacco RL, Homma S, Di Tullio MR. Association between diabetes mellitus and left ventricular hypertrophy in a multi-ethnic population. Am J Cardiol 2008;101(12):1787-91. https://doi.org/10.1016/j.amjcard.2008.02. 082.

16. Abdalla M, Booth JN, Diaz KM, Sims M, Muntner P, Shimbo D. Hypertension and alterations in left ventricular structure and geometry in African Americans: the Jackson Heart study. J Am Soc Hypertens 2016;10(7):550-558.e10. https://doi.org/10.1016/j. jash.2016.05.010.

17. Sarnak MJ, Levey AS, Schoolwerth AC, Coresh J, Culleton B, Hamm LL, et al. Kidney disease as a risk factor for development of cardiovascular disease: A statement from the American heart association councils on kidney in cardiovascular disease, high blood pressure research, clinical cardiology, and epidemiology and prevention. Hypertension 2003;42(5):1050-65. https://doi.org/10. 1161/01.CIR.0000095676.90936.80.

18. Maripov A, Mamazhakypov A, Sartmyrzaeva M, Akunov A, Uulu KM, Duishobaev M, et al. A. Right ventricular remodeling and dysfunction in obstructive sleep apnea: a systematic review of the literature and meta-analysis. Can Respir J 2017:1587865 https://d oi.org/10.1155/2017/1587865.

19. Kolb TM, Hassoun PM. Right ventricular dysfunction in chronic lung disease. Cardiol Clin 2012;30(2):243-56. https://doi.org/10. 1016/j.ccl.2012.03.005.

20. Frost A, Badesch D, Gibbs JSR, Gopalan D, Khanna D, Manes A, et al. Diagnosis of pulmonary hypertension. Eur Respir J 2019;53:1801904. https://doi.org/10.1183/13993003.01904-2018.

21. Galie N, Humbert M, Vachiery JL, Gibbs S, Lang I, Torbicki A, et al. 2015 ESC/ERS Guidelines for the diagnosis and treatment of pulmonary hypertension. Eur Respir J 2015;46(4):903-75.

22. Cox DR. Regression models and life-tables. J R Stat Soc Ser B 1972;34:187-220. https://doi.org/10.1007/978-1-4612-4380-9_37.

23. Sciagra R. Right ventricular perfusion: do we need additional evidence or just a simple methodology. J Nucl Cardiol 2019;26:272-4.

24. Burger IA, Husmann L, Herzog BA, Buechel RR, Pazhenkottil AP, Ghadri JR, et al. Main pulmonary artery diameter from attenuation correction CT scans in cardiac SPECT accurately predicts pulmonary hypertension. J Nucl Cardiol 2011;18(4):63441.

25. Mazraeshahi RM, Striet J, Oeltgen RC, Gerson MC. Myocardial SPECT images for diagnosis of pulmonary hypertension and right ventricular hypertrophy. J Nucl Med Technol 2010;38(4):175-80.

26. Khaja F, Alam M, Goldstein S, Anbe DT, Marks DS. Diagnostic value of visualization of the right ventricle using thallium-201 myocardial imaging. Circulation 1979;59:182-8.

27. Hirschfeld CB, Dondi M, Pascual TNB, Mercuri M, Vitola J, Karthikeyan G, et al. Worldwide diagnostic reference levels for single photon emission computed tomography myocardial perfusion imaging: findings from INCAPS. JACC Cardiovasc Imaging 2020;S1936-878X(20)30605-7.

28. Jouni H, Askew JW, Crusan DJ, Miller TD, Gibbons RJ. Temporal trends of single-photon emission computed tomography myocardial perfusion imaging in patients without prior coronary artery disease: A 22 year experience at a tertiary academic medical center. Am Heart J 2016;176:127-33.

29. Horwich TB, Fonarow GC, Hamilton MA, MacLellan WR, Woo MA, Tillisch JH. The relationship between obesity and mortality in patients with heart failure. J Am Coll Cardiol 2001;38(3):78995 .

30. Costanzo P, Cleland JGF, Pellicori P, Clark AL, Hepburn D, Kilpatrick ES, et al. The obesity paradox in type 2 diabetes mellitus: relationship of body mass index to prognosis: a cohort study. Ann Intern Med 2015;162(9):610-8.

31. Jose A, King CS, Shlobin OA, Brown W, Wang C, Nathan SD. Exercise pulmonary hemodynamic response predicts outcomes in fibrotic lung disease. Eur Respir J 2018;52(3):1801015.

32. Goransson C, Vejlstrup N, Carlsen J. Exercise cardiovascular magnetic resonance imaging allows differentiation of low-risk pulmonary arterial hypertension. J Heart Lung Transplant 2019;38(6):627-35

33. Jernigan DB. CDC COVID-19 response team. Update: public health response to the coronavirus disease 2019 outbreak-United States. Morb Mortal Wkly Rep 2020;69:216-9.

34. Baier AW, Snyder DJ, Leahy IC, Patak LS, Brustowicz RM. A shared opportunity for improving electronic medical record data. Anesth Analg 2017;125(3):952-7.

35. Gunnarsdottir OS, Rafnsson V. Mortality of the users of a hospital emergency department. Emerg Med J 2006;23(4):

36. Basu S, Berkowitz SA, Phillips RL, Bitton A, Landon BE, Phillis RS. Association of Primary Care Physician supply with population mortality in the united states, 2005-2015. JAMA Int Med 2019;179(4):506-14.

37. Blackstone EH. Demise of a vital resource. J Thorac Cardiovasc Surg 2012;143(1):37-8

38. Curtis MD, Griffith SD, Tucker M, Taylor MD, Capra WB, Carrigan $\mathrm{G}$, et al. Development and validation of a high-quality composite real-world mortality endpoint. Health Serv Res 2018;53(6):4460-76

39. Huston JH, Maron BA, French J, Huang S, Thayer T, Farber-Eger $\mathrm{EH}$, et al. Association of mild echocardiographic pulmonary hypertension with mortality and right ventricular function. JAMA Cardiol 2019;4(11):1112-21.

40. Kiatchoosakun S, Wongvipaporn C, Nanagara R, Hoit BD. Right ventricular systolic pressure assessed by echocardiography: a predictive factor of mortality in patients with scleroderma. Clin Cardiol 2011;34(8):488-93.

41. Wang N, Fulcher J, Abeysuriya N, McGrady M, Wilcox I, Celermajer D, et al. Tricuspid regurgitation is associated with increased mortality independent of pulmonary pressures and right heart failure: a systematic review and meta-analysis. Eur Heart J 2019;40(5):476-84.

Publisher's Note Springer Nature remains neutral with regard to jurisdictional claims in published maps and institutional affiliations. 